\title{
$\beta$ Cephei Stars from ASAS: a New Look at Hot Pulsators
}

\author{
Andrzej Pigulski* and Grzegorz Pojmański ${ }^{\dagger}$ \\ *Instytut Astronomiczny Uniwersytetu Wrocławskiego, Kopernika 11, 51-622 Wrocław, Poland \\ $\dagger$ Obserwatorium Astronomiczne Uniwersytetu Warszawskiego, Al. Ujazdowskie 4, 00-478 Warszawa, Poland
}

\begin{abstract}
The All Sky Automated Survey (ASAS) data, covering nearly $70 \%$ of the whole sky south of declination $+28^{\circ}$, were recently used to discover about $300 \beta$ Cephei stars brighter than $\mathrm{V} \sim 11.5 \mathrm{mag}$. As this means a nearly fourfold increase in the number of known stars of this type, new possibilities in studying these pulsators open up, including statistical work.

The homogeneity of the ASAS survey allows us to study their distribution in the local Galaxy. We discuss this distribution in the context of the location of nearby spiral arms. In addition, we show pulsational (periods, amplitudes) properties of the whole sample of known $\beta$ Cephei stars. Some objects interesting from the point of view of asteroseismology are also indicated.
\end{abstract}

Keywords: Time-series analysis; Pulsations, oscillations and stellar seismology; Low-amplitude blue variables ( $\beta$ Cephei) PACS: $95.75 . \mathrm{Wx}$, 97.10.Sj, 97.30.Dg

\section{INTRODUCTION}

The most recent review paper on $\beta$ Cephei stars, young, massive and hot main-sequence pulsators was presented several years ago by [1]. They provided a list of $93 \mathrm{mem}-$ bers of this group that were discovered during a centurylong history of their investigation. The review was followed by several comprehensive multi-site campaigns focused on selected $\beta$ Cephei stars, namely those that had the largest number of modes known: $v$ Eri, 12 (DD) Lac, V836Cen, and $\theta$ Oph. These observations allowed to make a reliable mode identification and consequently enabled seismic modeling.

In the last few years, the first satellite observations of $\beta$ Cephei stars were also conducted. Several bright stars of this type ( $\beta \mathrm{Cru}, \lambda \mathrm{Sco}, \kappa \mathrm{Sco}, \alpha \mathrm{Vir}, \beta \mathrm{CMa}$, $\alpha$ Lup, $v$ Eri, $\beta$ Cep) were observed by the WIRE satellite tracker [2]. One of the most interesting results of these observations was the confirmation of the eclipsing nature of $\lambda$ Sco [3]. Next, the Canadian MOST satellite observed several $\beta$ Cephei stars: $\delta$ Cet [4, 5, 6], $\gamma$ Peg [7] and Spica which was found to be eclipsing [8]. Finally, GSC 06272-01557 = ALS 4680 was discovered as a new $\beta$ Cephei star [9]. It was independently found in the ASAS data (see the next section), where only two strongest modes were detected. The third satellite that had $\beta$ Cephei stars in the target list was CoRoT. It observed V1449 Aql [10], the only $\beta$ Cephei star known in the CoRoT fields at the time of target selection.

During the last few years, some serendipitous discoveries as well as regular searches, especially in the young open clusters, increased the number of known $\beta$ Cephei stars by additional 19 objects. However, the largest impact on the number of known $\beta$ Cephei stars came from the All Sky Automated Survey (ASAS). In this paper, we show how this survey contributed to our knowledge of this group of pulsating stars.

\section{$\beta$ CEPHEI STARS FROM ASAS}

The ongoing, third part of the ASAS, ASAS-3 [11, 12], covers about 70 per cent of the sky south of declination $+28^{\circ}$. It started in 2000 and already brought the discovery of over 50000 variable stars published in the form of the catalog [13, 14, 15, 16, 17]. The stars in the catalog were classified by means of an automatic classification scheme that utilized mainly periods, amplitudes and near-infrared photometry of variable stars. The $\beta$ Cephei as a group was first included in the third paper of the series [15]. However, since $\beta$ Cephei stars are low-amplitude pulsators, the automatic classification was not always sufficient to distinguish unambiguously a $\beta$ Cephei star from the other low-amplitude short-period variable stars. Thus, this classification was verified by [18] who used additional information on the ASAS variables spread over the literature, mainly spectral types and/or $U B V$ photometry. In consequence, $14 \beta$ Cephei stars were found. In addition, [19] did the same for the ASAS-2 data [20] finding five more stars of this type.

All these nineteen $\beta$ Cephei stars found in the public ASAS data had relatively large amplitudes, namely larger than $\sim 30 \mathrm{mmag}$. This was the consequence of the method of the selection of objects for the ASAS variablestar catalogue, namely by means of the magnitudedispersion diagram. On the other hand, the quality and number of the ASAS data allowed detection of periodic signals with amplitudes down to a few mmag. An obvious conclusion was that many more $\beta$ Cephei stars could be discovered using the ASAS data. The search for these 


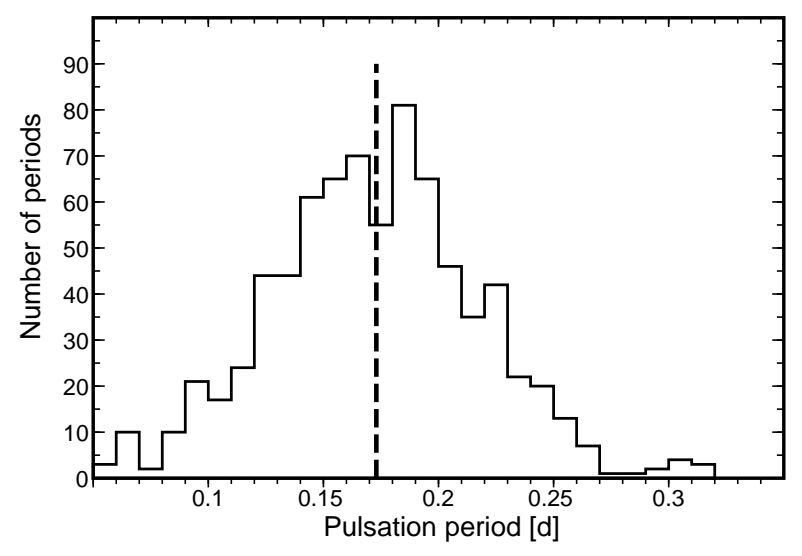

FIGURE 1. Period distribution for modes detected in 406 known $\beta$ Cephei stars. The vertical dashed line shows the mean and median value of the distribution equal to $0.173 \mathrm{~d}$.

variables was done in two steps. First, about $15000 \mathrm{O}$ and early B-type stars were selected using over two hundred catalogs with suitable information (mainly on spectral types and multi-colour photometry). Next, the data for the selected stars were analyzed in detail. The result largely exceeded the expectations: $276 \beta$ Cephei stars were discovered. The first part of this work was already published: [21] presented the list of 103 new $\beta$ Cephei stars. A detailed description of the remaining stars is in the course of preparation for publication.

In the following subsections we present the main properties of the whole group of $406 \beta$ Cephei stars that are currently known, dividing them on two parts: those discovered with the use of the ASAS data (295 stars) and the remaining 112 stars. For clarity, we will refer to these two groups as ASAS and non-ASAS $\beta$ Cephei stars, respectively.

\section{Pulsational properties}

The most important pulsational characteristics of $\beta$ Cephei stars include periods and amplitudes. In the whole group of over $400 \beta$ Cephei stars, we know over 750 independent pulsation modes. Distribution of their periods is shown in Fig. 10 The periods longer than $0.35 \mathrm{~d}$ were excluded from this figure. They are observed in hybrid $\beta$ Cephei/SPB pulsators and represent most likely $g$ modes. The distribution of periods is quite symmetric around the mean value of $0.173 \mathrm{~d}$ (shown with vertical dashed line in Fig. 1). The median value of the distribution is the same as the mean.

The distribution of $V$-filter semi-amplitudes for the same sample of modes is plotted against the period of the mode in Fig.2 In this figure, the data for ASAS and nonASAS $\beta$ Cephei stars are shown with different symbols.

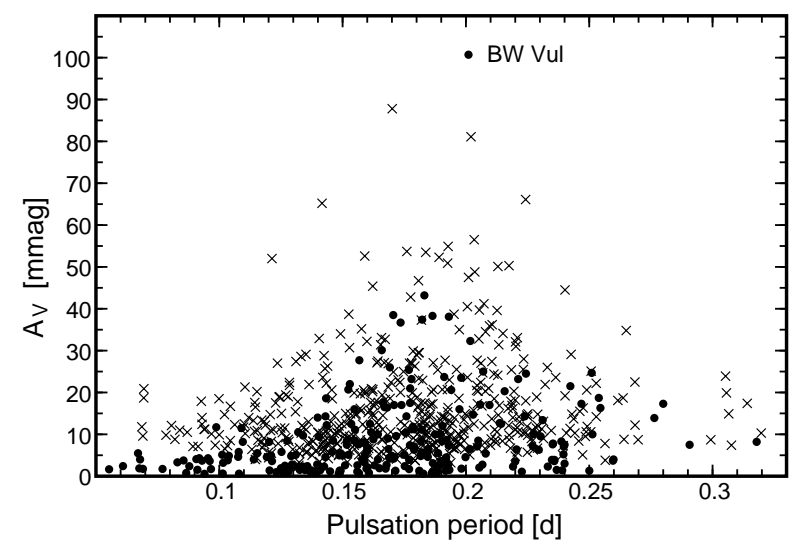

FIGURE 2. $V$-filter semi-amplitudes, $A_{V}$, for the same modes that are shown in Fig. 1 plotted against their pulsation periods. Amplitudes for the ASAS $\beta$ Cephei stars are shown as crosses, for the remaining stars, as dots. The star with the largest amplitude known, BW Vul, is labeled.

It can be clearly seen that most of the large-amplitude pulsators were found in the ASAS data, although the well-known variable BW Vul (labeled) remained the star with the largest amplitude known. Nevertheless, the gap between 40 and 100 mmag has been filled up with the modes detected in the ASAS $\beta$ Cephei stars. With so many modes we may also safely conclude that $0.1 \mathrm{mag}$ is an observational upper limit for the $V$-filter semiamplitude of a $\beta$ Cephei star. Another feature that can be seen from Fig. 2 is that stars with the largest amplitudes are found in the middle of the range of periods observed in $\beta$ Cephei stars. Close to the limits of this range, the amplitudes tend to be smaller. This can only be explained in view of the non-linear theory of pulsations and the results of the first theoretical works [22] seem to agree with the observed distribution.

From the point of view of asteroseismology, the best are those stars that have both large amplitudes (mode identification is easier for them) and many modes excited. It is even better if some modes are rotationally split which can be recognized by the presence of multiplets equidistant or almost equidistant in frequency. [21] presented a list of seven stars with likely rotational splittings. In the second group of analyzed stars, two more stars with almost equidistant triplets, ALS 2932 and ALS 9911, were found.

Another group of stars that can be very useful in seismic modeling are hybrid $\beta$ Cephei/SPB stars, i.e. stars that exhibit both $p$ (high-frequency) modes intrinsic to $\beta$ Cephei stars and $g$ (low-frequency) modes characteristic for the SPB stars. Several candidates for hybrid $\beta$ Cephei/SPB stars among the ASAS $\beta$ Cephei stars were indicated by [21]. Again, some more were found in the second group. One of them, HDE 313073, is es- 


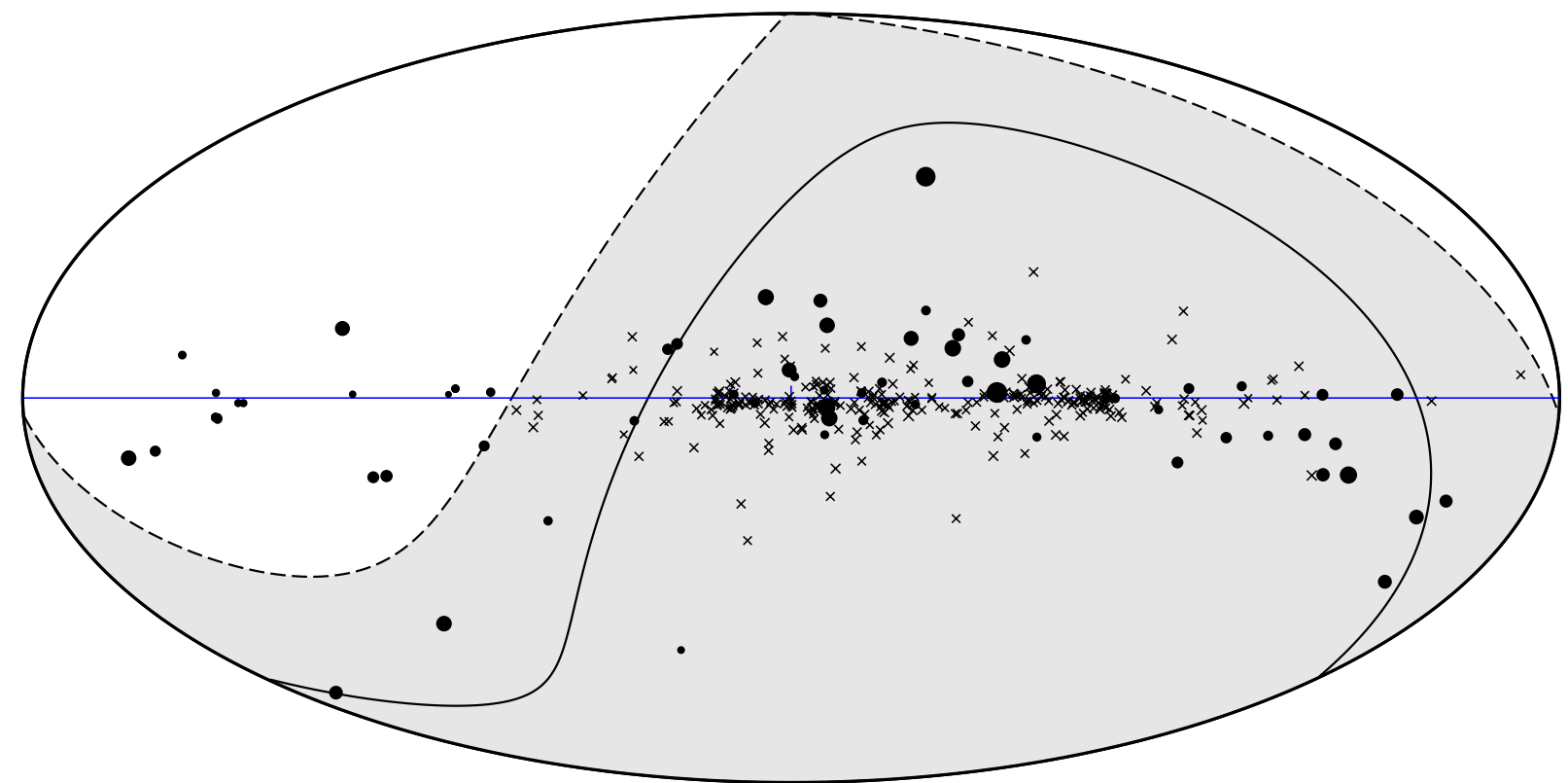

FIGURE 3. Distribution of the ASAS (crosses) and non-ASAS $\beta$ Cephei stars (filled circles) in Galactic coordinates shown in Aitoff projection. The size of the circle depends on the magnitude of a star: brighter stars are shown with larger circles. The Galactic center is located in the middle of the oval. The sky covered by the ASAS observations $\left(\delta<+28^{\circ}\right)$ is shown in gray and delimited by a dashed line. Solid line is the celestial equator.

pecially interesting as it shows two modes in the lowfrequency $\left(0.4-0.5 \mathrm{~d}^{-1}\right)$ and two modes in the highfrequency $\left(7.2-7.4 \mathrm{~d}^{-1}\right)$ domain. It is interesting to note that the ASAS candidates for hybrids have, at least for the low-frequency modes, much larger amplitudes than those found for such modes in the bright hybrids $12 \mathrm{Lac}$, $v$ Eri, and $\gamma$ Peg [7].

\section{Distribution in the sky and the Galaxy}

Distribution of the ASAS and non-ASAS $\beta$ Cephei stars in the sky is shown in Fig. 3. Since $\beta$ Cephei stars are young objects, their concentration towards the Galactic plane is obvious. On the other hand, the ASAS $\beta$ Cephei stars do not spread uniformly along the Galactic plane. They are located mainly at galactic longitudes, $l$, between $285^{\circ}$ and $20^{\circ}$ with a lower density for $310^{\circ}<l<330^{\circ}$. The clumping is partially due to the non-uniform extinction, but the main effect is a consequence of the young age of these stars. Since young stars are distributed largely close to the star-forming regions, they trace the location of spiral arms. For this reason, so few $\beta$ Cephei stars were discovered for $l<285^{\circ}$, where there is no nearby spiral arm. This can be better seen in Fig. 4, where the ASAS $\beta$ Cephei stars were projected onto the Galactic plane. The distances of stars were estimated using the $U B V$ photometry in the same way as in [18]. As can be seen, most of the stars have distances in the range of 1-3 kpc and they are located in the Sagittarius-Carina spiral arm. However, stars up to the distance of $5 \mathrm{kpc}$ are observed. Some of them might be runaway stars, which are located at high Galactic latitudes as can be seen in Fig. 3 .

\section{CONCLUSIONS}

The ASAS provided a large number of bright $\beta$ Cephei stars and many of them are good targets for asteroseismology. Their $V$ magnitudes range between 8 and 12 mag. This is a very convenient range for the followup photometric and spectroscopic work. They are neither too bright for photometry with a small telescope nor too faint for spectroscopy with a moderate-size one. In addition, many of them have large amplitudes and are multiperiodic. The problem is that the ASAS $\beta$ Cephei stars are mostly southern objects and a very limited number of sites with the appropriate telescopes is currently available. The discovery, however, might be a very good reason for preserving small telescopes in the southern sites and devote them to the follow-up photometry and/or spectroscopy of $\beta$ Cephei stars.

The ASAS survey was recently extended to the Northern hemisphere [12]. Thus, a similar analysis will be soon possible for the whole northern sky. In particular, 


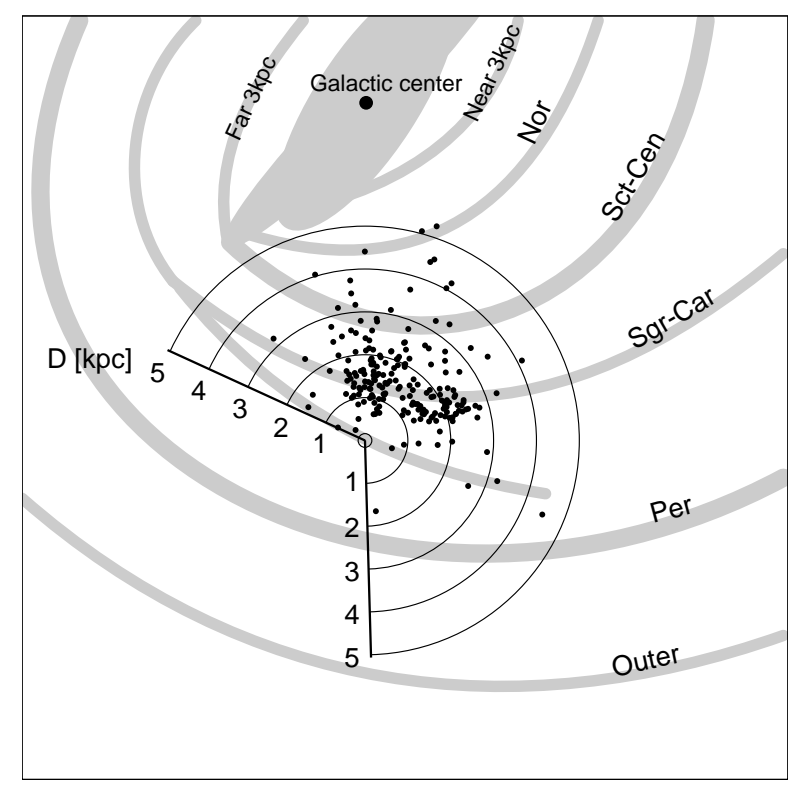

FIGURE 4. The ASAS $\beta$ Cephei stars (dots) projected onto the Galactic plane. The Sun is located in the center of the arcs that cover the range of galactic longitudes corresponding to the part of the sky observed by the ASAS. The location of spiral arms was adopted following [23]; they were labeled with common names. The short, unlabeled arm is the Orion Spur.

we may expect discovering a large number of $\beta$ Cephei stars located in the Perseus arm (see Fig. 4). It must be noted, however, that due to the poor spatial resolution of the ASAS frames, the ASAS photometry in very dense regions is not very good. Consequently, the ASAS $\beta$ Cephei stars are mostly field objects or objects located in sparse open clusters and associations. The searches for these stars in young open clusters, some ongoing (see, e.g. [24] and references therein), can be therefore stated as a good complementary work.

\section{ACKNOWLEDGMENTS}

This work was supported by the grant N N203 302635 from Polish MNiSzW.

\section{REFERENCES}

1. A. Stankov, and G. Handler, ApJS 158, 193-216 (2005).

2. H. Bruntt, Comm. in Asteros. 150, 326-332 (2007).

3. H. Bruntt, and D.L. Buzasi, Mem. S.A.It. 77, 278-281 (2006).

4. C. Aerts, S.V. Marchenko, J.M. Matthews, et al., ApJ 642, 470-477 (2006).

5. M. Jerzykiewicz, Acta Astronomica 57, 33-47 (2007).

6. R. Moldovan, J.M. Matthews, and C. Cameron, these proceedings (2009).
7. G. Handler, J.M. Matthews, J.A. Eaton, et al., ApJ, 698, L56-L59 (2009).

8. M. Desmet, G.A.H. Walker, S. Yang, et al., these proceedings (2009).

9. V. Antoci, G. Handler, R. Kuschnig, M. Hareter, and J.M. Matthews, these proceedings (2009).

10. P. Degroote, M. Briquet, C. Catala, et al., astro$\mathrm{ph} / 0906.4057$ (2009).

11. G. Pojmański, A.S.P. Conf. Ser., 246, 53-58 (2001).

12. G. Pojmański, A.S.P. Conf. Ser., 403, 52-70 (2009).

13. G. Pojmański, Acta Astronomica, 52, 397-427 (2002).

14. G. Pojmański, Acta Astronomica, 53, 341-369 (2003).

15. G. Pojmański, and G. Maciejewski, Acta Astronomica, 54, 153-179 (2004).

16. G. Pojmański, and G. Maciejewski, Acta Astronomica, 55, 97-122 (2005).

17. G. Pojmański, B. Pilecki, and D. Szczygieł, Acta Astronomica, 55, 275-301 (2005).

18. A. Pigulski, Acta Astronomica, 55, 219-236 (2005).

19. G. Handler, Inf. Bull. Var. Stars, 5667, 1-5 (2005).

20. G. Pojmański, Acta Astronomica, 50, 177-190 (2000).

21. A. Pigulski, and G. Pojmański, $A \& A, 477,917-929$ (2008).

22. R. Smolec, and P. Moskalik, MNRAS, 377, 645-656 (2007).

23. E. Churchwell, L. Babler, M.R. Meade, et al., PASP, 121, 213-230 (2009).

24. Z. Kołaczkowski, A. Pigulski, G. Kopacki, and G. Michalska, Acta Astronomica, 54, 33-55 (2004). 